\title{
Neural Concomitants of Remote Memory in a Comedian with Exceptional Verbal Memory
}

\author{
Nathalie Mella ${ }^{1}$ (D) Frédéric Grouiller ${ }^{2} \cdot$ Michael Mouthon $^{3} \cdot$ Fabienne Picard $^{4}$ \\ Received: 15 September 2020 / Accepted: 4 March 2021 / Published online: 20 March 2021 \\ (C) The Author(s) 2021
}

\begin{abstract}
Most studies exploring how remote memory is represented in the brain are based on strong episodic self-related components. Because of methodological reasons, much less is known about how the information concerning the semantic part of autobiographical memory is retrieved, and whether the brain correlates differ according to the autobiographical moment of the memory formation. In the present study, we explored the neural concomitants of the retrieval of texts learnt at different periods of life, in a comedian with exceptional verbal memory skills. This 49-year-old comedian was instructed to recite aloud a total of 30 texts he learnt during three different epochs: before the age of 15 years (E1), between the age of 15 and 25 years (E2), and after the age of 25 years (E3). The most salient activation was observed for memory from the farthest period, with a preponderance of the medial rostral prefrontal cortex (PFC) and of the precuneus. There was no hippocampal activation during text retrieval by comparison to a control condition, whatever the learning period. This study supports the assumption that the recall of remote semantic memories can occur without hippocampal activation. We discussed the activation of the rostral PFC during retrieval of the oldest (and best consolidated) memories as the possible involvement of control meta-memory processes rather than memory processes per se.
\end{abstract}

Keywords Semantic memory $\cdot$ Retrieval $\cdot$ Rostral prefrontal cortex $\cdot$ Remote memory $\cdot$ Hippocampus

\section{Introduction}

Autobiographical memory, the recollection of episodes from an individual's life, is composed of both episodic (personal experiences that are specific in time and place) and semantic (general knowledge about the self-related world) memories (Levine et al., 2002; Tulving, 2002). While the recall of episodic memory along different periods of life has been extensively studied, this is much less true for semantic memory, and the process of consolidation of semantic memory into long-

Nathalie Mella

Nathalie.mella-barraco@unige.ch

1 Department of Psychology, University of Geneva, Geneva, Switzerland

2 Department of Radiology and Medical Informatics, University Hospitals of Geneva and Faculty of Medicine, Geneva, Switzerland

3 Neurology Unit, Department of Medicine, Faculty of Sciences, University of Fribourg, Fribourg, Switzerland

4 Department of Neurology, University Hospitals and Medical School of Geneva, Geneva, Switzerland term memory is still being the subject of much debate (Sekeres et al., 2017).

There is a converging evidence that the hippocampus has a time-limited role in the acquisition and storage of semantic knowledge: the hippocampus appears to be essential for the formation and maintenance of semantic memory for a period of a few years after learning, while, as time passes, increasing involvement of multiple neocortical, especially frontal regions, was demonstrated (Smith \& Squire, 2009). Regarding the crucial involvement of the frontal cortex in the recall of more remote memories, the hypothesis that older memories require more strategic, effortful search has been raised. Theories of memory consolidation, such as the multi-trace transformation theory (Moscovitch et al., 2006; Sekeres et al., 2018) or more recently the contextual binding theory (Yonelinas et al., 2019), posit that the hippocampus is necessary to the recall of rich detailed memories, but not for the retrieval of less contextualized information, such as semantic memories.

Most studies exploring remote memory are based on strong episodic self-related components (Bonnici et al., 2012; Bonnici et al., 2013; Steinvorth et al., 2005; Tomadesso 
et al., 2015). Much less is known about how the information concerning the semantic part of autobiographical memory is retrieved, and whether the brain correlates differ according to the autobiographical moment of the memory formation. A major difficulty for non-episodic autobiographical settings is the failure to sample adequately the early years of life, e.g., difficulty in many studies to assert that items from remote time periods were in fact learnt remotely (Squire \& Bayley, 2007). In the present report, we were able to overcome these difficulties by examining performance of a professional comedian who started to learn texts in the early childhood, and who is able to exactly settle texts acquisition in different periods of his life. This case thus represents a unique opportunity to better understand how semantic memories are represented in the brain according to the moment of acquisition in life. We conducted a functional magnetic resonance imaging (fMRI) experiment to investigate the cerebral activation underlying the retrieval of semantic memories in this subject with exceptional verbal memory skills, comparing brain activation for texts learnt in three different periods of his lifetime.

\section{Material and Methods}

\section{Subject}

The 49-year-old right-handed professional French comedian (AC) of the present study has developed exceptional verbal memory skills. From the age of 5 years until today, he has performed 1 -h daily memory training. He would be able to recite up to $30 \mathrm{~h}$ of different texts (i.e., 30.000 lines). As a comedian, the subject regularly recites a variety of these texts in his theater performances. He indicated that he could clearly distinguish a "different memory" related to 3 distinct periods of learning (epochs): between the age of 5 and 15 years, between the age of 15 and 25 years, and after the age of 25 years. He asserted that the texts he learnt before the age of 15 could be recited easily without any risk of error and without previous review. However, the texts he learnt later needed to be read again once (for texts learnt between 15 and 25 years old) or several times (for the texts learnt after 25 years old) before a performance. In addition, as concerns the episodic aspects of these memories, AC reports being able to set the learning of the texts in a temporal context (e.g., the year of learning), and consequently to remind the global location (e.g., the city he lived in). Very few details are associated to the learning of the texts. If these memories have then intertwined episodic and semantic components, they pertain more to the semantic part of autobiographical memory. The subject gave his written informed consent, and the study was approved by the local ethical committee of the Geneva University Hospital.

\section{Memory Task}

Prior to the experimental sessions, the participant gave 30 titles of texts for different periods of learning: (E): E1, before the age of 15 years (10 texts); E2, between the age of 15 and 25 years (10 texts); and E3, after the age of 25 years (10 texts). He chose them among a big amount of texts he had learned up to the study.

During the fMRI, the subject was instructed to recite aloud the text corresponding to the title written on the screen. Texts were presented in a pseudo-random temporal order. As a control condition (CTRL), the subject was asked to recite in loop the days of the week. Each text (or control condition) was recited for $36 \mathrm{~s}$, before the occurrence of another instruction, and was considered as a "block". Two sessions of 15 minutes were performed. Each session consisted of 25 blocks: 5 blocks of each epoch (E1, E2, and E3), 5 blocks of control condition, and 5 blocks of resting (fixation cross). The 25 blocks were pseudo-randomized.

Instructions (titles of the texts, "weekdays", or fixation cross) were presented for $2 \mathrm{~s}$ on a screen visible through a mirror mounted on the head coil. In case of hesitation or memory lapse, the subject was instructed to continue further or to start again from the beginning of the text. The correct accomplishment of the task was controlled by the use of a noise cancelation MR-compatible audio recording system.

\section{MRI Acquisition}

The experiment was performed in a $3 \mathrm{~T}$ whole-body MRI (twelve-channel head receive coil with body transmit coil, Siemens Magnetom Trio, Erlangen, Germany). Two runs of 460 functional images were acquired using a single-shot $\mathrm{T}_{2}{ }^{*}$ weighted gradient-echo echo-planar imaging (EPI) sequence $\left(\mathrm{TR}=2000 \mathrm{~ms}, \mathrm{TE}=30 \mathrm{~ms}\right.$, flip angle $=85^{\circ}$, voxel size $=2 \times$ $2 \times 3.85 \mathrm{~mm}^{3}, 29$ slices). To correct images for geometric distortions induced by local magnetic field inhomogeneity, a B0 fieldmap was obtained from two gradient echo sequence $(\Delta \mathrm{TE}=2.46 \mathrm{~ms}) . \mathrm{A} 3 \mathrm{D}$ high-resolution $\mathrm{T}_{1}$-weighted structural image was also acquired for anatomical localization.

\section{Analyses}

The fMRI data analysis was performed using SPM8 software (Wellcome Department of Imaging Neuroscience, UCL, London, UK). Spatial preprocessing of functional images included: (i) realignment and unwarping of the fMRI time series (Andersson et al., 2001); (ii) rigid-body coregistration of the 3D T1 structural image on the realigned functional images (Ashburner \& Friston, 1997); (iii) normalization to the Montreal Neurological Institute (MNI) space using normalization parameters estimated by $3 \mathrm{D}$ T1 segmentation 
(Ashburner \& Friston, 2005) and resampling of the normalized functional images to $2 \times 2 \times 2 \mathrm{~mm}^{3}$; and (iv) spatial smoothing with an isotropic Gaussian kernel $(6 \mathrm{~mm}$ full width at the half maximum).

Finally, fMRI time series were whitened and serial correlations were modeled using an auto-regressive filter of order 1. Low-frequency noise and signal drift were removed using a discrete cosine transform (DCT) basis set with a filter cut-off period of $256 \mathrm{~s}$.

Each condition (E1, E2, E3, control, and resting) was modeled as a boxcar function convolved with the canonical hemodynamic response function. The time periods when the subject was hesitating or had a memory lapse were included into the model as confounds. Twenty-four motion-related parameters (six realignment parameters with a Volterra expansion) were also included into the model as covariates (Friston et al., 1996) to remove any residual motion-related variance. Statistical analyses of fMRI data were performed using a mass-univariate approach based on the general linear model (Friston et al., 1994).

We tested the differences between each learning epoch separately against the control condition (E1>CTRL, E2 $>$ CTRL and E3>CTRL) and between each learning epoch $(\mathrm{E} 1>\mathrm{E} 3, \mathrm{E} 1>\mathrm{E} 2, \mathrm{E} 2>\mathrm{E} 3, \mathrm{E} 2>\mathrm{E} 1, \mathrm{E} 3>\mathrm{E} 1, \mathrm{E} 3>\mathrm{E} 2)$ using onesample $t$ test. The statistical significance level of the resulting SPM T-maps was set to $p<0.05$ corrected for multiple comparisons across the whole brain using family-wise error (FWE) correction. A 5-voxel extent threshold was applied.

The anatomical localization of each activated cluster (Table 1) is determined using the automated anatomical labeling (AAL) SPM-toolbox, which is based on the anatomical parcellation of the MNI brain template into 90 regions (Tzourio-Mazoyer et al., 2002).

Regions of interest analyses were performed specifically on left and right hippocampi using MarsBaR toolbox (Brett et al., 2002).

\section{Results}

Details of the significantly activated areas for each condition are given in Table 1.

\section{fMRI Activations for each Epoch}

The activations obtained during each epoch against the control condition (E1>CTRL, E2>CTRL, E3>CTRL) are presented in Fig. 1. The activations were wider in the two latest periods (E2 and E3). The middle frontal gyrus, more specifically the medial part of the rostral prefrontal cortex (PFC) (approximately BA10), was significantly activated for each epoch (all $p<.001$, random-field (FWE) corrected for multiple comparisons). Additionally the remembering of texts learnt in the two latest periods activated bilateral inferior frontal cortex, left superior frontal cortex, bilateral parietal cortex, and left supplementary motor cortex. There was no visible hippocampal or para-hippocampal activation during retrieval of texts, whatever the learning period, after subtracting the activation obtained with recitation of the weekdays (control condition). Regions of interest analyses on hippocampi confirmed the absence of significant activation in left ( $p=0.984, p=$ 0.965 , and $p=0.960$, for E1, E2, and E3, respectively) and right ( $p=0.969, p=0.579$, and $p=0.847$, for $\mathrm{E} 1, \mathrm{E} 2$, and $\mathrm{E} 3$, respectively) hippocampi.

\section{Differences Between Epochs}

The most salient activation is observed when subtracting the activation generated by retrieval of texts from epoch E3 (after the age of 25 years) from that of epoch E1 (before the age of 15 years), with a specific activation in the medial part of the rostral PFC and in the precuneus (Fig. 2) (the random-field (FWE) corrected $p$-value at the cluster size is $p<0.001$ for the precuneus and $p=0.005$ for the PFC).

It must be underlined that the activation of the rostral PFC was higher during retrieval of texts from the second learning period (E2, 15-25 years) than during the first period (E1, under 15 years) or during the third period (E3, after 25 years). However, this activation observed for the texts learnt during the second period was associated with a very large activation of many other cortical areas, while the activation was quite isolated in the rostral PFC for the texts learnt during the oldest period, corresponding to the texts with the most consolidated memory.

\section{Discussion}

The present study intended to explore the cerebral correlates of memory for texts learnt at different life periods in a single subject showing outstanding verbal memory skills that he worked daily since the age of 5 . The major result was a specific activation of the bilateral rostral PFC and the right precuneus for the retrieval of the most remote semantic memory. In addition, no hippocampal or entorhinal activation was observed during the retrieval of texts of any of the three life periods.

Neuropsychological and fMRI studies suggest that the hippocampus is involved in both recent and remote memory as far as episodic memory is concerned (Bonnici et al., 2012). The role of mesiotemporal structures, especially the hippocampus, in long-term semantic memory is less clear. In a study of patients with bilateral hippocampal damage, the remote memory for factual knowledge from 11 to 30 years before amnesia was intact (Manns et al., 2003). In healthy subjects, Smith and Squire showed a progressive decrease in activity in 
Table 1 List of activated clusters and their anatomical localization for each contrast $(p<0.05$, family-wise error correction for multiple comparisons, 5 voxel extent cluster).

\begin{tabular}{|c|c|c|c|c|}
\hline Contrast & $\begin{array}{l}\text { MNI coordinates } \\
{[\mathrm{x} ; \mathrm{y} ; \mathrm{z}]}\end{array}$ & $\mathrm{T}$ & $\mathrm{k}$ & Regions \\
\hline \multirow[t]{6}{*}{ E1 - CTRL } & {$[30 ; 58 ;-10]$} & 6.64 & 23 & Right middle frontal gyrus, orbital part \\
\hline & {$[-8 ; 56 ;-8]$} & 6.24 & 98 & Left middle frontal gyrus, orbital part \\
\hline & {$[22 ; 50 ;-14]$} & 6.17 & 18 & Left middle frontal gyrus, orbital part \\
\hline & {$[30 ; 52 ; 0]$} & 5.42 & 16 & Left middle frontal gyrus, orbital part \\
\hline & {$[8 ; 60 ;-16]$} & 5.41 & 6 & Right gyrus rectus \\
\hline & {$[-52 ;-28 ;-8]$} & 5.28 & 6 & Left middle temporal gyrus \\
\hline \multirow[t]{31}{*}{ E2 - CTRL } & {$[30 ; 60 ; 10]$} & 9.06 & 1249 & Right middle frontal gyrus, orbital part \\
\hline & {$[-30 ; 52 ; 4]$} & 8.99 & 1542 & Left middle frontal gyrus, orbital part \\
\hline & {$[62 ; 4 ;-6]$} & 7.78 & 83 & Right insula \\
\hline & {$[-54 ;-2 ; 42]$} & 7.66 & 315 & Left middle frontal gyrus, lateral part \\
\hline & {$[-64 ;-44 ; 20]$} & 7.49 & 220 & Left middle temporal gyrus \\
\hline & {$[-52 ;-28 ;-8]$} & 7.13 & 58 & Left middle temporal gyrus \\
\hline & {$[-40 ; 12 ; 26]$} & 7.00 & 91 & Left inferior frontal gyrus (P. triangularis) \\
\hline & {$[58 ;-2 ; 40]$} & 6.97 & 55 & Right postcentral gyrus \\
\hline & {$[-2 ; 8 ; 64]$} & 6.85 & 104 & Left supplementary motor area \\
\hline & {$[22 ; 48 ;-14]$} & 6.78 & 30 & Right middle frontal gyrus, orbital part \\
\hline & {$[-18 ; 6 ; 14]$} & 6.67 & 51 & Left putamen \\
\hline & {$[48 ; 36 ; 26]$} & 6.42 & 28 & Right inferior frontal gyrus (P. triangularis) \\
\hline & {$[-10 ; 30 ; 32]$} & 6.19 & 21 & Left anterior cingulate \\
\hline & {$[30 ;-68 ; 46]$} & 6.11 & 51 & Right superior parietal lobule \\
\hline & {$[58 ;-20 ;-8]$} & 6.01 & 14 & Right middle temporal gyrus \\
\hline & {$[66 ;-12 ;-8]$} & 5.94 & 6 & Right middle temporal gyrus \\
\hline & {$[-54 ;-16 ; 38]$} & 5.87 & 30 & Left postcentral gyrus \\
\hline & {$[42 ; 42 ;-14]$} & 5.82 & 8 & Right inferior frontal gyrus (P. orbitalis) \\
\hline & {$[-58 ;-22 ;-2]$} & 5.71 & 12 & Left middle temporal gyrus \\
\hline & {$[-60 ;-16 ; 8]$} & 5.66 & 8 & Left superior temporal gyrus \\
\hline & {$[64 ; 0 ; 18]$} & 5.61 & 10 & Right postcentral gyrus \\
\hline & {$[66 ;-26 ;-2]$} & 5.59 & 14 & Right middle temporal gyrus \\
\hline & {$[62 ;-8 ; 2]$} & 5.51 & 10 & Right superior temporal gyrus \\
\hline & {$[-48 ;-38 ; 52]$} & 5.51 & 15 & Left inferior parietal lobule \\
\hline & {$[-54 ; 20 ; 26]$} & 5.49 & 5 & Left inferior frontal gyrus (P. triangularis) \\
\hline & {$[-62 ;-2 ; 0]$} & 5.40 & 15 & Left middle temporal gyrus \\
\hline & {$[-8 ; 52 ; 32]$} & 5.33 & 17 & Left superior frontal gyrus, medial part \\
\hline & {$[-36 ; 24 ; 42]$} & 5.32 & 13 & Left middle frontal gyrus, lateral part \\
\hline & {$[48 ;-28 ;-4]$} & 5.29 & 6 & Right superior temporal gyrus \\
\hline & {$[-38 ;-16 ; 60]$} & 5.27 & 8 & Left precentral gyrus \\
\hline & {$[-60 ; 10 ; 16]$} & 5.09 & 5 & Left inferior frontal gyrus (P. opercularis) \\
\hline \multirow[t]{10}{*}{ E3 - CTRL } & {$[-46 ;-4 ; 54]$} & 8.97 & 248 & Left middle frontal gyrus, lateral part \\
\hline & {$[-64 ;-44 ; 20]$} & 8.12 & 171 & Left middle temporal gyrus \\
\hline & {$[-54 ; 20 ; 26]$} & 7.68 & 180 & Left inferior frontal gyrus (P. triangularis) \\
\hline & {$[-14 ; 54 ;-18]$} & 7.50 & 108 & Left middle frontal gyrus, orbital part \\
\hline & {$[8 ; 60 ;-18]$} & 7.12 & 30 & Right superior frontal gyrus, medial orbital part \\
\hline & {$[22 ; 48 ;-16]$} & 6.74 & 23 & Right middle frontal gyrus, orbital part \\
\hline & {$[-6 ; 16 ; 48]$} & 6.10 & 43 & Left superior frontal gyrus, medial part \\
\hline & {$[-50 ;-28 ;-8]$} & 6.04 & 13 & Left middle temporal gyrus \\
\hline & {$[-42 ; 30 ; 38]$} & 6.03 & 25 & Left middle frontal gyrus, lateral part \\
\hline & {$[-2 ; 8 ; 62]$} & 5.93 & 24 & Left supplementary motor area \\
\hline
\end{tabular}


Table 1 (continued)

\begin{tabular}{|c|c|c|c|c|}
\hline Contrast & $\begin{array}{l}\text { MNI coordinates } \\
{[\mathrm{x} ; \mathrm{y} ; \mathrm{z}]}\end{array}$ & $\mathrm{T}$ & $\mathrm{k}$ & Regions \\
\hline & {$[48 ; 36 ; 26]$} & 5.91 & 12 & Right inferior frontal gyrus (P. triangularis) \\
\hline & {$[60 ;-4 ; 40]$} & 5.79 & 25 & Right postcentral gyrus \\
\hline & {$[30 ;-64 ; 44]$} & 5.75 & 21 & Right angular gyrus \\
\hline & {$[-32 ; 50 ; 4]$} & 5.60 & 59 & Left middle frontal gyrus, lateral part \\
\hline & {$[30 ; 58 ; 10]$} & 5.43 & 21 & Right middle frontal gyrus, lateral part \\
\hline & {$[-60 ; 2 ; 26]$} & 5.32 & 9 & Left precentral gyrus \\
\hline & {$[34 ; 48 ;-2]$} & 5.22 & 7 & Right middle frontal gyrus, orbital part \\
\hline \multirow[t]{2}{*}{ E1 - E3 } & {$[6 ;-54 ; 68]$} & 5.99 & 29 & Right precuneus \\
\hline & {$[6 ; 66 ;-10]$} & 5.78 & 7 & Right superior frontal gyrus, medial orbital part \\
\hline \multirow[t]{3}{*}{ E2 - E3 } & {$[-8 ; 62 ; 0]$} & 6.57 & 113 & Left superior frontal gyrus, medial orbital part \\
\hline & {$[6 ; 66 ; 2]$} & 5.90 & 75 & Right superior frontal gyrus, medial orbital part \\
\hline & {$[6 ;-54 ; 66]$} & 4.97 & 6 & Right precuneus \\
\hline
\end{tabular}

$T$ maximal $T$ value for the cluster; $k$ number of activated voxels in the cluster

the hippocampus for recalled memory from 1 to 12 years prior, and then stable low activity for more remote memories (Smith \& Squire, 2009), while other studies pointed out that semantic memories can exist independently of the hippocampus (Moscovitch et al., 2006). According to theories of memory consolidation, such as the multi-trace transformation theory (Moscovitch et al., 2006; Sekeres et al., 2018) and the contextual binding theory (Yonelinas et al., 2019), the hippocampus is part of a retrieval network for recent memories, and has only a limited role in the retrieval of remote semantic memories. Once consolidated in long-term semantic memory, successful retrieval of remote events would rely more on strengthened interconnections between and within neocortical regions than on the entorhinal cortex (Haist et al., 2001; Sekeres et al., 2018; Yonelinas et al., 2019). In our study, the recall of remote semantic memories was not dependent on the hippocampus, for any of the three periods. Within theories of memory consolidation, our result suggests that the texts learnt by A.C. at the three different periods of life are accessible out of their context of learning and with no rich details. Altogether, it supports the idea that these very remote semantic memories have been progressively decontextualized because they have been repeatedly remembered and the learning context has lost in vividness and precision, becoming more and more self-unrelated.

In our subject, access to the most remote memories selectively activated the medial rostral PFC and the precuneus. Several studies have reported significant stronger activity for remote hits than recent hits in the medial PFC (Frankland \& Bontempi, 2005; Gais et al., 2007), and shown medial PFC activation when recollecting remote (autobiographical) memory after long delays (between 2 and 30 years) (Cabeza \& St Jacques, 2007; Svoboda et al., 2006). In agreement with these findings, the medial PFC was the only known brain region in rodents where a circumscribed lesion caused a selective memory deficit for remote but not recent memories (Takehara et al.,

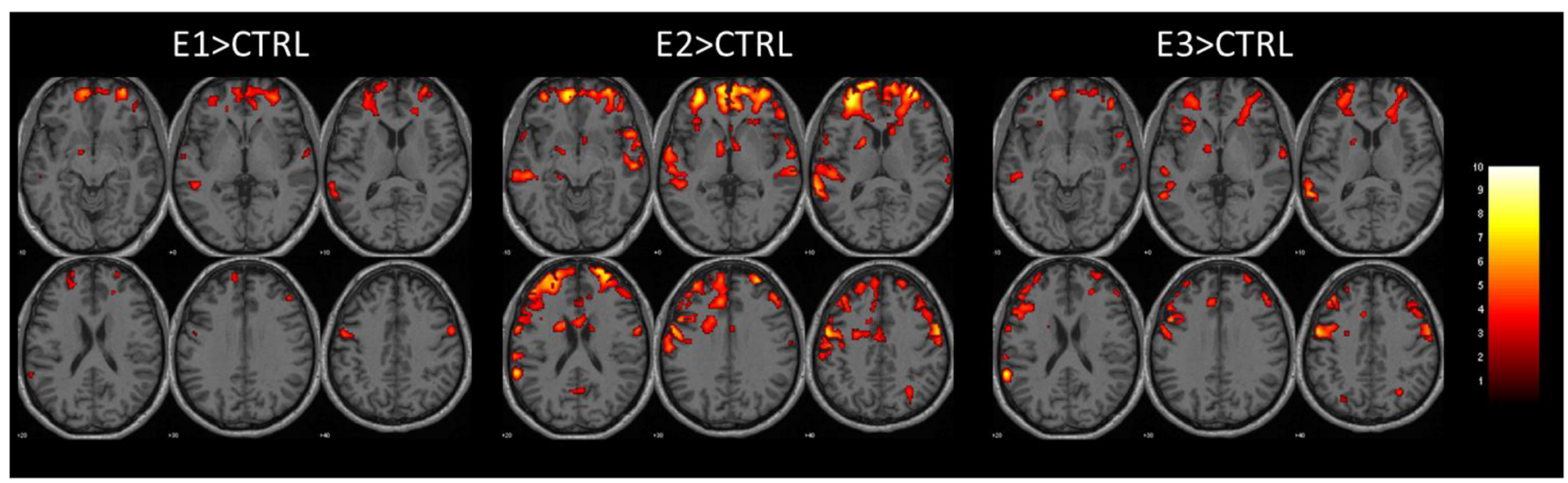

Fig. 1 Activations obtained during recitation of texts learnt during each epoch (E1: before the age of 15 years, E2: between the ages of 15 and 25, E3: after the age of 25). Bilateral ventromedial prefrontal cortex
(VMPFC) (or medial orbitofrontal cortex) is activated for each epoch $(p$ $<0.001$, uncorrected) 
Fig. 2 Activation of bilateral anterior ventromedial prefrontal cortex (VMPFC) $(T=5.78)$ and right precuneus $(T=5.99) \mathrm{ob}-$ tained from the contrast $\mathrm{E} 1>\mathrm{E} 3(p$ $<0.001$, uncorrected)
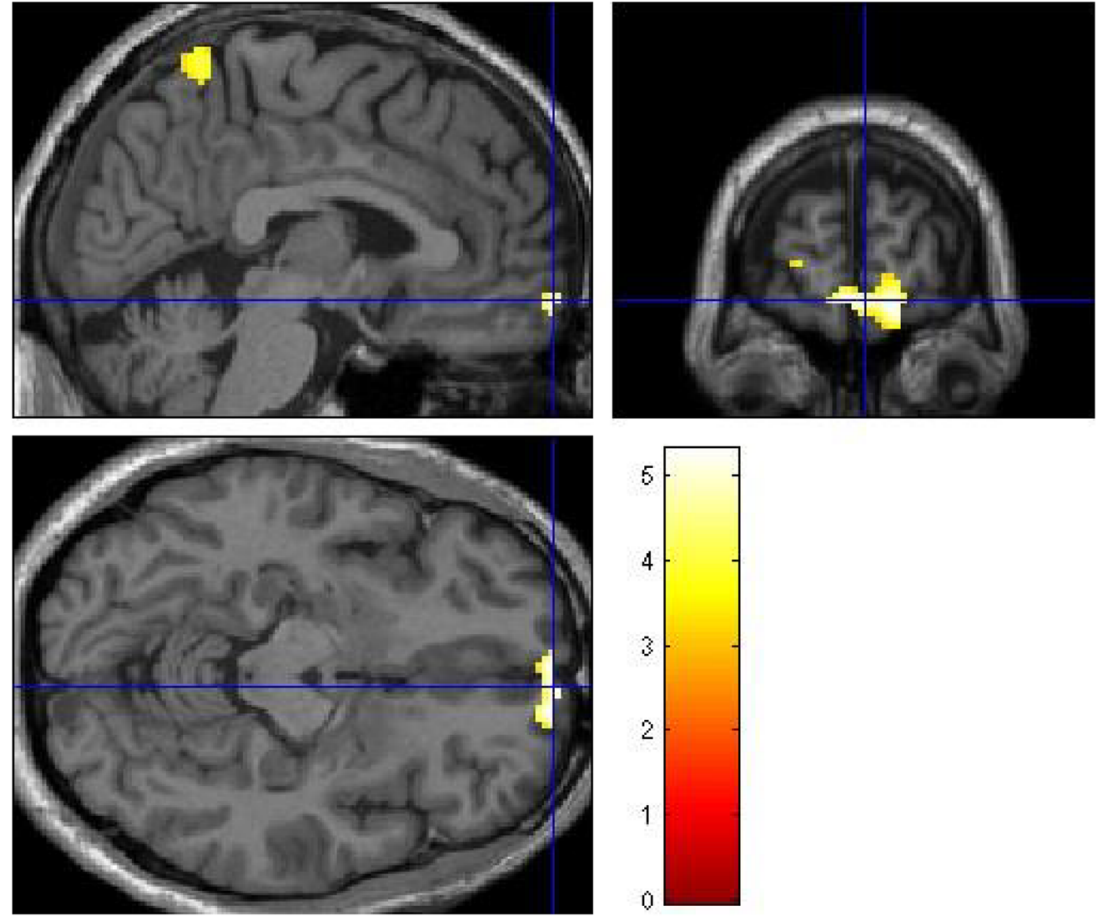

2003). The selective activation of the medial part of the rostral PFC for the most remote memory in our subject is interesting regarding the "gateway hypothesis" proposed by Burgess et al. (2007). According to Burgess et al. (2007), the anterior part of the rostral PFC would be involved in low demand task processing, serving as a "fast route" for content scheduling. Studies showing a negative link between reaction times and activation of the medial rostral PFC support this assumption (Gilbert et al., 2006). The anterior PFC has been more largely involved in metacognitive judgments of memory (direct appraisal of memory contents and of the strength of memory traces) (Baird et al., 2013; Nelson, 1990). The study of Baird et al. (2013) supported a role of the anterior PFC in the monitoring of cognition and experience, with the medial subdivision assessing data from memory stored over long time scales. The medial anterior PFC was shown to be involved in metacognitive evaluation of memory retrieval such as "feeling of knowing" judgments (Schnyer et al., 2005) or retrospective confidence ratings (Modirrousta \& Fellows, 2008; Moritz et al., 2006). Medial PFC lesions are associated with impairments in appraisal of memory (Modirrousta \& Fellows, 2008; Pannu \& Kaszniak, 2005). In summary, the rostral PFC, and particularly its medial part, would be involved more in memory control processes, such as information content scheduling, than in memory retrieval processes per se.

Following the above-mentioned concepts, the observed activation of the most anterior part of rostral PFC in our subject might index high meta-memory capacities and a lower level of difficulty in reciting remote and well-anchored texts than in reciting texts learnt in his later periods of life. This assumption fits well with the subjective reports of the subject of this study, attesting that texts learnt in infancy can be recited almost automatically and with a great facility, by comparison to texts learnt later in life, which have to be read once or more before a performance. The selective activation of the rostral PFC in the recitation of texts firmly anchored in our subject's long-term memory suggests the involvement of meta-memory processes rather than of memory processes per se. Going further and considering our subject's extremely high memory capacities, we may hypothesize that he has developed strong metamemory competencies. More generally, our result may suggest that the development of powerful memory capacities does not rely upon the development of memory system per se but rather on increased competencies of attentional monitoring systems, involved in meta-memory processes. Further group studies should confirm this assumption.

Our results also revealed greater activation of the precuneus during the recitation of texts learnt during childhood than during the recitation of texts acquired later in life. This structure is thought to be involved in several high-order cognitive functions, including visuospatial imagery, episodic memory retrieval, and self-related processes (see Cavanna \& Trimble, 2006 for a review). Interestingly, in memory tasks, greater activity in the precuneus has been linked to older versus new correct judgments in memory for words (Henson et al., 2000), although in this study old and new words were learnt in the same day. Other reports in the literature suggest that activation in the precuneus may be linked to familiarity processes in memory recollection (Yonelinas et al., 2005). More recently, Baird et al. (2013) and McCurdy et al. (2013) 
elegantly demonstrated the metacognitive role of the precuneus in memory tasks. The metacognitive ability for evaluations of memory indeed correlated with an increased connectivity between medial anterior PFC and the right precuneus (and intraparietal sulcus). A role of the precuneus in mnemonic metacognition was also recently shown through an alteration of its activity with repetitive transcranial stimulation in healthy people (Ye et al., 2018).

Besides, the volume of the precuneus correlates with better meta-memory abilities (McCurdy et al., 2013). This volume was positively linked with the degree of confidence in correctness in a verbal memory task. It was also significantly related to similar metacognitive judgments (correctness) in a visual task, suggesting a role of this structure in general metacognitive functions.

To sum up, the present experiment gave the opportunity to explore the cerebral correlates of access to memories learnt at different periods of life in a single subject, based on the retrieval of texts from three remote learning life periods. Our results support models of memory consolidation positing that access to remote consolidated memories does not necessarily require the hippocampus. Following these models, it suggests that these repeatedly remembered memories have been progressively decontextualized, losing their detailed and vivid episodic components to become almost exclusively semantic. The specific activation of the rostral PFC and precuneus for the retrieval of the most remote and well-anchored texts suggests the implication of attentional metacognitive processes rather than of memory processes per se in the recollection of these texts. More generally, considering the temporal gradient of our subject's confidence in his memory performance, i.e., the oldest the memory the better the remembering and the identified regions of activation, we hypothesize that mnemonic metacognitive processes play a major role in long-term memory retrieval skills. As this is a single case study, future studies using paradigms applicable to group designs will be necessary to further explore this issue.

Acknowledgments We thank A.C. for his kind participation in the study. We thank an anonymous reviewer for helpful comments improving the paper. We also thank Prof. Martial Van der Linden, Prof. Armin Schnider, Dr. Pasquale Calabrese, Dr. Frédéric Assal and Dr JeanMarie Annoni for helpful comments and fruitful discussions. This work has been supported by the Center for Biomedical Imaging (CIBM, Director: Prof. François Lazeyras) of the Geneva-Lausanne Universities, the EPFL (Lausanne, Switzerland) and the GenevaLausanne University Hospitals.

Data and Material Availability Data will be available upon request.

Code Availability Not applicable.

Authors' Contributions NM wrote the paper, FG analyzed the data, MM performed the experiment, and FP conceived the experiment and wrote the paper.
Funding Open Access funding provided by Université de Genève.

\section{Declarations}

Ethics Approval/Consent to Participate This study was designed and conducted in accordance with the Helsinki declaration. The participant gave informed consent to participate in this study.

Consent to Publish AC, the participant, signed informed consent regarding publishing his data and photographs.

Consent for Publication All authors agreed to submit the final version of this manuscript.

Conflicts of Interest The authors declare no competing interests.

Open Access This article is licensed under a Creative Commons Attribution 4.0 International License, which permits use, sharing, adaptation, distribution and reproduction in any medium or format, as long as you give appropriate credit to the original author(s) and the source, provide a link to the Creative Commons licence, and indicate if changes were made. The images or other third party material in this article are included in the article's Creative Commons licence, unless indicated otherwise in a credit line to the material. If material is not included in the article's Creative Commons licence and your intended use is not permitted by statutory regulation or exceeds the permitted use, you will need to obtain permission directly from the copyright holder. To view a copy of this licence, visit http://creativecommons.org/licenses/by/4.0/.

\section{References}

Andersson, J. L., Hutton, C., Ashburner, J., Turner, R., \& Friston, K. (2001). Modeling geometric deformations in EPI time series. Neuroimage, 13(5), 903-919. https://doi.org/10.1006/nimg.2001. 0746.

Ashburner, J., \& Friston, K. (1997). Multimodal image coregistration and partitioning-a unified framework. Neuroimage, 6(3), 209-217. doi: S1053-8119(97)90290-1 [pii]. https://doi.org/10.1006/nimg.1997.0290.

Ashburner, J., \& Friston, K. J. (2005). Unified segmentation. Neuroimage, 26(3), 839-851. doi: S1053-8119(05)00110-2 [pii]. https://doi.org/10.1016/j.neuroimage.2005.02.018.

Baird, B., Smallwood, J., Gorgolewski, K. J., \& Margulies, D. S. (2013). Medial and lateral networks in anterior prefrontal cortex support metacognitive ability for memory and perception. Journal of Neuroscience, 33(42), 16657-16665.

Bonnici, H. M., Chadwick, M. J., Lutti, A., Hassabis, D., Weiskopf, N., \& Maguire, E. A. (2012). Detecting representations of recent and remote autobiographical memories in vmPFC and hippocampus. The Journal of Neuroscience, 32(47), 16982-16991.

Bonnici, H. M., Chadwick, M. J., \& Maguire, E. A. (2013). Representations of recent and remote autobiographical memories in hippocampal subfields. Hippocampus, 23(10), 849-854.

Brett, M., Anton, J.-L., Valabregue, R., \& Poline, J.-B. (2002). Region of interest analysis using an SPM toolbox. Paper presented at the 8th international conference on functional mapping of the human brain.

Burgess, P. W., Dumontheil, I., \& Gilbert, S. J. (2007). The gateway hypothesis of rostral prefrontal cortex (area 10) function. Trends in Cognitive Sciences, 11(7), 290-298.

Cabeza, R., \& St Jacques, P. (2007). Functional neuroimaging of autobiographical memory. Trends in Cognitive Sciences, 11(5), 219- 
227. doi: S1364-6613(07)00078-2 [pii]. https://doi.org/10.1016/j. tics.2007.02.005.

Cavanna, A. E., \& Trimble, M. R. (2006). The precuneus: A review of its functional anatomy and behavioural correlates. Brain, 129(3), 564-583.

Frankland, P. W., \& Bontempi, B. (2005). The organization of recent and remote memories. Nature Reviews. Neuroscience, 6(2), 119-130. https://doi.org/10.1038/nrn1607.

Friston, K. J., Holmes, A. P., Worsley, K. J., Poline, J. P., Frith, C. D., \& Frackowiak, R. S. J. (1994). Statistical parametric maps in functional imaging: A general linear approach. Human Brain Mapping, 2(4), 189-210. https://doi.org/10.1002/hbm.460020402.

Friston, K. J., Williams, S., Howard, R., Frackowiak, R. S., \& Turner, R. (1996). Movement-related effects in fMRI time-series. Magnetic Resonance in Medicine, 35(3), 346-355 Retrieved from http:// www.ncbi.nlm.nih.gov/pubmed/8699946.

Gais, S., Albouy, G., Boly, M., Dang-Vu, T. T., Darsaud, A., Desseilles, M., et al. (2007). Sleep transforms the cerebral trace of declarative memories. Proceedings of the National Academy of Sciences of the United States of America, 104(47), 18778-18783. https://doi.org/ 10.1073/pnas.0705454104.

Gilbert, S. J., Simons, J. S., Frith, C. D., \& Burgess, P. W. (2006). Performance-related activity in medial rostral prefrontal cortex (area 10) during low-demand tasks. Journal of Experimental Psychology: Human Perception and Performance, 32(1), 45.

Haist, F., Bowden Gore, J., \& Mao, H. (2001). Consolidation of human memory over decades revealed by functional magnetic resonance imaging. Nature Neuroscience, 4(11), 1139-1145. https://doi.org/ 10.1038/nn739nn739 [pii].

Henson, R., Rugg, M., Shallice, T., \& Dolan, R. J. (2000). Confidence in recognition memory for words: dissociating right prefrontal roles in episodic retrieval. Journal of Cognitive Neuroscience, 12(6), 913 923

Levine, B., Svoboda, E., Hay, J. F., Winocur, G., \& Moscovitch, M. (2002). Aging and autobiographical memory: Dissociating episodic from semantic retrieval. Psychology and Aging, 17(4), 677.

Manns, J. R., Hopkins, R. O., \& Squire, L. R. (2003). Semantic memory and the human hippocampus. Neuron, 38(1), 127-133 doi: S0896627303001466 [pii].

McCurdy, L. Y., Maniscalco, B., Metcalfe, J., Liu, K. Y., de Lange, F. P., \& Lau, H. (2013). Anatomical coupling between distinct metacognitive systems for memory and visual perception. The Journal of Neuroscience, 33(5), 1897-1906.

Modirrousta, M., \& Fellows, L. K. (2008). Medial prefrontal cortex plays a critical and selective role in 'feeling of knowing'meta-memory judgments. Neuropsychologia, 46(12), 2958-2965.

Moritz, S., Gläscher, J., Sommer, T., Büchel, C., \& Braus, D. F. (2006). Neural correlates of memory confidence. Neuroimage, 33(4), 1188 1193.

Moscovitch, M., Nadel, L., Winocur, G., Gilboa, A., \& Rosenbaum, R. S. (2006). The cognitive neuroscience of remote episodic, semantic and spatial memory. Current Opinion in Neurobiology, 16(2), 179-190. doi: S0959-4388(06)00038-9 [pii]. https://doi.org/10. 1016/j.conb.2006.03.013.

Nelson, T. O. (1990). Metamemory: A theoretical framework and new findings. In Psychology of learning and motivation (Vol. 26, pp. 125-173). Elsevier.
Pannu, J. K., \& Kaszniak, A. W. (2005). Metamemory experiments in neurological populations: A review. Neuropsychology Review, 15(3), 105-130.

Schnyer, D. M., Nicholls, L., \& Verfaellie, M. (2005). The role of VMPC in metamemorial judgments of content retrievability. Journal of Cognitive Neuroscience, 17(5), 832-846.

Sekeres, M. J., Moscovitch, M., \& Winocur, G. (2017). Mechanisms of memory consolidation and transformation. In Cognitive neuroscience of memory consolidation (pp. 17-44). Berlin: Springer.

Sekeres, M. J., Winocur, G., \& Moscovitch, M. (2018). The hippocampus and related neocortical structures in memory transformation. Neuroscience Letters, 680, 39-53.

Smith, C. N., \& Squire, L. R. (2009). Medial temporal lobe activity during retrieval of semantic memory is related to the age of the memory. The Journal of Neuroscience, 29(4), 930-938. https:// doi.org/10.1523/JNEUROSCI.4545-08.2009.29/4/930 [pii].

Squire, L. R., \& Bayley, P. J. (2007). The neuroscience of remote memory. Current Opinion in Neurobiology, 17(2), 185-196. doi: S09594388(07)00030-X [pii]. https://doi.org/10.1016/j.conb.2007.02.006.

Steinvorth, S., Levine, B., \& Corkin, S. (2005). Medial temporal lobe structures are needed to re-experience remote autobiographical memories: Evidence from HM and WR. Neuropsychologia, 43(4), 479-496.

Svoboda, E., McKinnon, M. C., \& Levine, B. (2006). The functional neuroanatomy of autobiographical memory: A meta-analysis. Neuropsychologia, 44(12), 2189-2208. doi: S00283932(06)00209-0 [pii]. https://doi.org/10.1016/j.neuropsychologia. 2006.05.023.

Takehara, K., Kawahara, S., \& Kirino, Y. (2003). Time-dependent reorganization of the brain components underlying memory retention in trace eyeblink conditioning. The Journal of Neuroscience, 23(30), 9897-9905 doi:23/30/9897 [pii].

Tomadesso, C., Perrotin, A., Mutlu, J., Mézenge, F., Landeau, B., Egret, S., et al. (2015). Brain structural, functional, and cognitive correlates of recent versus remote autobiographical memories in amnestic Mild Cognitive Impairment. NeuroImage: Clinical, 8, 473-482.

Tulving, E. (2002). Episodic memory: From mind to brain. Annual Review of Psychology, 53(1), 1-25.

Tzourio-Mazoyer, N., Landeau, B., Papathanassiou, D., Crivello, F., Etard, O., Delcroix, N., et al. (2002). Automated anatomical labeling of activations in SPM using a macroscopic anatomical parcellation of the MNI MRI single-subject brain. Neuroimage, 15(1), 273-289. https://doi.org/10.1006/nimg.2001.0978 S1053811901909784 [pii].

Ye, Q., Zou, F., Lau, H., Hu, Y., \& Kwok, S. C. (2018). Causal evidence for mnemonic metacognition in human precuneus. Journal of Neuroscience, 38(28), 6379-6387.

Yonelinas, A. P., Otten, L. J., Shaw, K. N., \& Rugg, M. D. (2005). Separating the brain regions involved in recollection and familiarity in recognition memory. The Journal of Neuroscience, 25(11), 30023008.

Yonelinas, A. P., Ranganath, C., Ekstrom, A. D., \& Wiltgen, B. J. (2019). A contextual binding theory of episodic memory: Systems consolidation reconsidered. Nature Reviews Neuroscience, 20(6), 364-375.

Publisher's Note Springer Nature remains neutral with regard to jurisdictional claims in published maps and institutional affiliations. 